\title{
Salvia hunzikeri (Lamiaceae), una nueva especie de los Andes del Perú
}

\section{Salvia hunzikeri (Lamiaceae), a new species from the Andes of Peru}

\section{Arturo Granda Paucar}

Facultad de Ciencias-Herbario, Universidad Nacional Agraria La Molina, Apdo. 456, Lima, Perú. Email: a.granda@lycos.com

$\begin{array}{ll}\text { Presentado: } & 01 / 05 / 2010 \\ \text { Aceptado: } & 05 / 10 / 2010\end{array}$ $\begin{array}{ll}\text { Aceptado: } & 05 / 10 / 2010 \\ \text { Publicado online: } & 14 / 12 / 2010\end{array}$

\section{Resumen}

Salvia hunzikeri (Lamiaceae), una nueva especie restringida al valle del río Mantaro en el Perú, es descrita, ilustrada y sus rasgos más notables contrastados con los de otras especies de la sección Flocculosae.

Palabras clave: Lamiaceae, Perú, Salvia, sección Flocculosae, subgénero Calosphace.

\section{Abstract}

Salvia hunzikeri (Lamiaceae), a new species restricted to Mantaro River valley in Peru, is described, illustrated, and its salient features contrasted to other species of section Flocculosae.

Keywords: Lamiaceae, Peru, Salvia, section Flocculosae, subgenus Calosphace.

\section{Introducción}

Moriré y no habré visto mi interminable casa J.L. Borges, El Perú

Salvia L. (Lamiaceae, Nepetoideae) es un género de áreas mayormente montano tropicales con un patrón fitogeográfico subcosmopolita manifiestamente afín al de Scutellaria L. (Paton 1990). A despecho de ser considerado un grupo natural debido a sus estructuras estaminales altamente especializadas, Walker et al. (2004), y posteriormente Walker \& Sytsma (2007), demostraron que Salvia es polifilético en tanto género, que sus miembros constituirían tres linajes, contenidos todos en el llamado 'clado Salvia' junto con otros cinco géneros actualmente reconocidos, y que su peculiar androceo habría evolucionado en paralelo al menos en tres ocasiones diferentes.

Dentro de los grupos infragenéricos tradicionalmente aceptados en Salvia, el subgénero Calosphace, ahora parte integrante de 'Salvia clado II' en términos de Walker et al. (2004), se erige como el grupo más rico del género, y en los confines andinosudamericanos, particularmente en territorio peruano, ha tenido gran oportunidad para llevar a cabo una significativa especiación en una amplia variedad de hábitats generados por la topografía y el clima, la que, y así lo sugiere la información cromosómica (Harley 1992), parece haberse visto fuertemente favorecida por la poliploidía. Asimismo, el origen y diversificación de las especies se vincula a la presencia de síndromes florales sofisticados necesarios para la transferencia del polen, que incluyen el bien conocido sistema estaminal de palanca, y que reflejan las relaciones exclusivas de mutualismo con los polinizadores como resultado a su vez de un fenómeno coevolutivo (Meeuse 1992, Claßen-Bockhoff et al. 2003, Wester \& Claßen-Bockhoff 2007).

En un conjunto tan amplio, cuya única revisión es debida a Epling (1939), no es extraña la reinterpretación de algunas de sus especies ni sorpresivo el hallazgo de nuevas entidades al paso que el análisis de un mayor número de especímenes de herbario corra parejas con estudios de campo más detallados (e. g. Fernández-Alonso 2003, Wood \& Harley 1989, Wood 2007).

Tras la correcta evaluación de sus particularidades, se describe a continuación una nueva especie de Salvia del Perú. El material estudiado, aunque escaso, indudablemente se diferencia de las especies conocidas y conviene no demorar más su publicación.

\section{Descripción}

Salvia hunzikeri A. Granda, sp. nov.

\section{Figura 1 A-J}

Tipo: Perú. Dpto. Huancavelica. Prov. Tayacaja, Hacienda Alalay, entre Mariscal Cáceres y Pampas, 3200-3250 m s.m., 13-IV-1953, O. Tovar 1367 (holotipo MOL, isotipo USM).

Suffrutex 0,4-0,6 $m$ altus, ramis ascendentibus, pilis ramosis floccoso-tomentellis demum glabratis; petiolis (3-)5-7 mm longis, foliorum laminis (20-)40-45 mm longis x 6-10 mm latis, lanceolatis vel elliptico-lanceolatis, in basi cuneatis, marginibus remote obscure crenato-serratis, in apice acutis vel acuminatis, paginis ambobus pilis ramosis et glandulis subsessilibus vestitis, superiore incana vel subglabra, inferiore dense viscido-flocculosa, venis prominulis; floribus (2-)6 in verticillastris, bracteis lanceolatis caducis subtentis; calycibus florentibus $(8,5-) 9-10$ $\mathrm{mm}$ longis, in maturitate $15 \mathrm{~mm}$ longis, extus pilis glandulosis subsessilibus et ramosis flocculosis; corollarum caerulearum, tubo 10-11 mm longo, leniter ventricoso, labia superiore 4,5-5 mm longa, inferiore 9-10 mm longa; staminibus in labia superiore galeata inclusis; stylo ad apicem utrimque hirsuto; nuculis (2,8-)3 mm longis, ellipticis.

Sufrútice de 0,4-0,6 m de altura, con ramas, hojas, pedicelos y cálices revestidos por indumento de tricomas multicelulares dendriformes, blanquecinos o algo ocráceos, con paredes gruesas y verrucosas, y glandulares subsésiles. Ramas arcuado-ascendentes de 0,5-2,0 mm diám., subteretes, parduscas a grisáceas, ligeramente estriadas, flocosas cuando jóvenes, las viejas glabrescentes, áfilas y nodosas, con las bases peciolares persistentes, internodos de (7-)10-40 mm long.; axilas foliares con innovaciones o fascículos de hojas correspondientes a las ramitas nuevas. Hojas con pecíolos de (3-)5-7 mm long., canaliculados, floculosotomentosos; láminas de (20-)40-45 mm long. x 6-10 mm lat., lanceoladas o también elíptico-lanceoladas, con base cuneada, márgenes ligeramente revolutos, tenuemente crenado-aserrados en su $1 / 2$ ó $3 / 4$ distales, ápice agudo o acuminado, concoloras o el haz apenas más obscuro, superficie adaxial moderadamente incana a glabrescente, abaxialmente con pubescencia más densa, algo víscida, floculosa a lo largo de los nervios, nerviación 


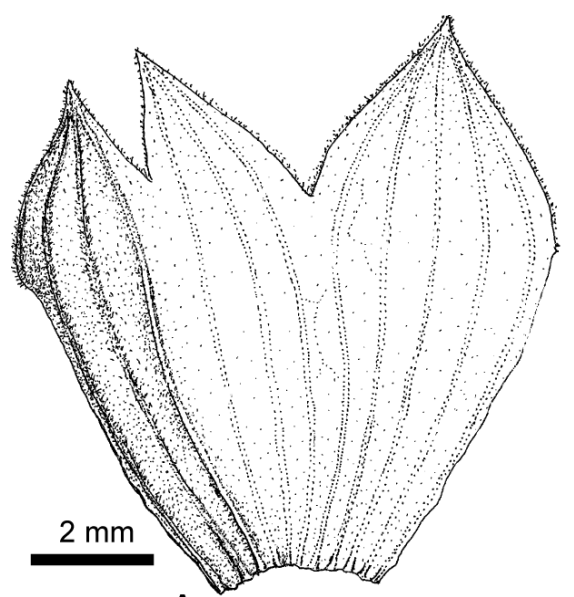

A

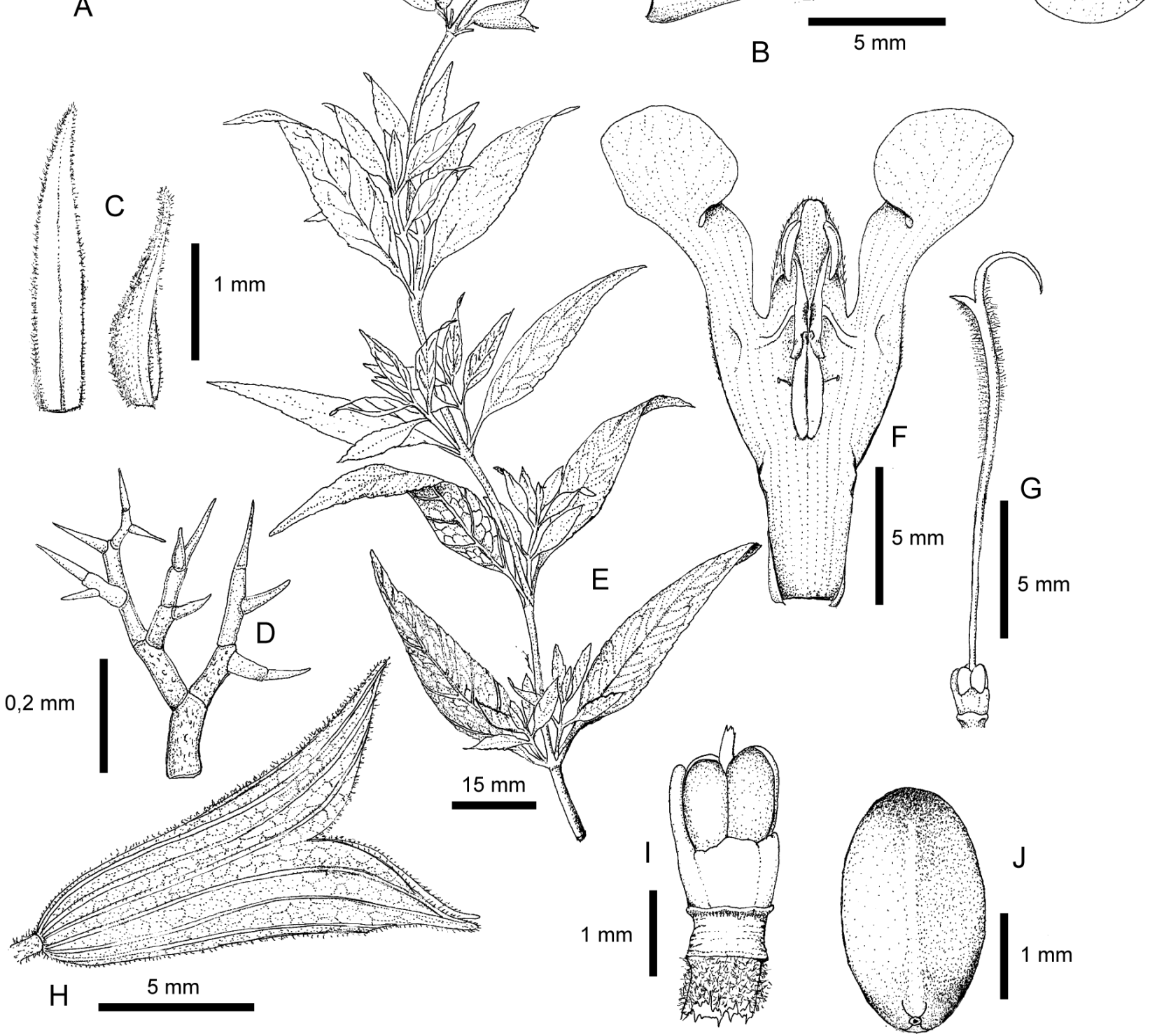

Figura 1. Salvia hunzikeri. A, Cáliz desplegado. B, Corola. C, Brácteas floríferas en vistas dorsal (indumento parcialmente removido) y lateral, respectivamente. D, Tricoma dendriforme calicinal. E, Rama florífera. F, Corola desplegada y androceo. G, Gineceo sobre el disco. H, Cáliz fructífero. I, Ovario y disco. J, Clusa en vista ventral. A-J, de O. Tovar 1367 (MOL).

semicraspedodrómica, con (5-)7-9 pares de nervios secundarios laterales, ascendentes, en general la nerviación impresa en el haz, el envés con los nervios principales eminentes y el retículo prominente. Flores reunidas en falsos racimos terminales cortos, de hasta $10 \mathrm{~cm}$ long., solitarios, verticilastros $5-7$, los inferiores distanciados por entrenudos de 10-15(-20) mm long. -a veces el basal en las axilas del par folioso distal-y los superiores más o menos confluentes, típicamente 6-floros o bien reducidos a 4- o incluso 2-floros en los nudos apicales; raquis similar al resto de las ramas; brácteas de $2-3 \mathrm{~mm}$ long. x $0,5-0,7 \mathrm{~mm}$ lat., muy prontamente caducas, lanceoladas o lineal-lanceoladas, semicóncavas, albiflocosas exteriormente, glabriúsculas en el interior; pedicelos de 2-5 $\mathrm{mm}$ long., ascendentes, flocosos, articulados al cáliz, tenaces. Cáliz en antesis de (8,5-)9-10 mm long., elongán- 
dose en fruto hasta $15 \mathrm{~mm}$ long., infundibuliforme, verdoso o con el dorso azul-violáceo, externamente floculoso, indumento algo más denso a lo largo de los nervios y en los márgenes de los labios, superficie interior con pubescencia antrorsa en sus 2/3 distales, labios distanciados en 4,7-5 mm long. y hasta en $9,5 \mathrm{~mm}$ en fruto; labio superior de 3,5-3,7 mm long. $\mathrm{x} 3,5-4,3$ $\mathrm{mm}$ lat., entero, ovado-deltoide, 7-nerviado, de ápice agudo; labio inferior de 3,2-3,3 mm long., 2-dentado, dientes de 2-2,3 mm long. $x$ 1,7-2 mm lat. en su sector libre, triangular-ovados, acuminados en el ápice y fusionados cerca de su $1 / 3$ basal u ocasionalmente hasta su mitad. Corola cerúlea de (17-)18-20 mm long., tubo de 10-11 mm long., excediendo brevemente al cáliz, recto o ligeramente curvo, un tanto ventricoso (hasta de $4 \mathrm{~mm}$ lat.), esparcidamente viloso en su mitad distal, los pelos simples y septados, glabro y epapiloso en el interior; labio superior de 4,5-5 mm long., cóncavo, emarginado, viloso; el inferior de 9-10 mm long., 3-lobado, viloso hacia la base, en ocasiones los pelos simples entremezclados con pelos ramificados, lobo medio mayor, de 5-6 mm long. x 8-9,5 mm lat., 2-lobulado. Estambres inclusos, insertos a $9 \mathrm{~mm}$ del borde basal de la corola, en las inmediaciones de las fauces, filamentos de ca. $2 \mathrm{~mm}$ long., prolongados en un diente corto en la articulación con el conectivo, polinatorios de $2,2 \mathrm{~mm}$ long., atenuados, vecciarios de 4,3 mm long. o uno algo menor, aplanados, conniventes hasta poco más arriba de su mitad -con diente retrorso de 0,3-0,5 mm long.-, papilosos a lo largo de sus márgenes internos (la línea de connivencia), esparcidamente cubiertos por pelitos capitados en sus caras dorsales e inmediaciones del diente. Tecas elipsoidales de 2,5 mm long. Estaminodios 2, de ca. $1 \mathrm{~mm}$ long., insertos a $8 \mathrm{~mm}$ por encima de la base del tubo. Disco con el lóbulo inferior prolongado, más o menos de la misma longitud que los lóbulos del ovario. Estilo de $13 \mathrm{~mm}$ long., aplanado lateralmente, ensanchándose suavemente hacia la zona de bifurcación, con pubescencia hirsuta cubriendo la mitad superior, más abundante en los márgenes, extendida por las ramas estigmáticas que son exertas y desiguales, la posterior mayor, de 4,5 mm long., subulada, algo recurvada, la inferior de 1-1,2 mm long., aguda y canaliculada. Clusas de (2,8-)3 mm long. x 1,7 mm lat., elipsoidales, obscuramente trígonas, castañas, superficie microscópicamente rugosa, aparentemente sin carácter mixocárpico.

Etimología.- Esta especie está dedicada a Armando T. Hunziker (1919-2001). A su legado de disciplina y capacidad de trabajo.

Distribución geográfica y hábitat.- Por ahora confinada a solo una localidad en la provincia de Tayacaja, departamento de Huancavelica, en elevaciones de 3200 - 3250 m de altitud. Weberbauer (1945) atribuye a esta zona del valle interandino del Mantaro un paisaje prevaleciente de estepas de gramíneas y arbustos bajos dispersos; sin embargo, la zona en cuestión parece ser de transición entre tal vegetación y una formación, común entre las laderas secas a niveles inferiores, con predominio de arbustos y arbolillos caducifolios asociados a gramíneas y herbáceas estacionales así como algunos elementos subxerófilos (Tovar 1957). Convive con especies tales como: Cosmos peucedanifolius Wedd., Cronquistianthus urubambensis (B.L. Rob.) R.M. King \& H. Rob., Gnaphalium dombeyanum DC., Oritrophium hieracioides (Wedd.) Cuatrec., Stevia puberula Hook., Coreopsis sp., Paranephelius sp., Calceolaria scabra Ruiz \& Pav., Escallonia myrtilloides L. f., Hesperoxiphion peruvianum (Baker) Baker,
Clinopodium breviflorum (Benth.) Govaerts, Agalinis sp., Agrostis tolucensis Kunth, Bromus lanatus Kunth, Thalictrum decipiens Boivin, Alonsoa acutifolia Ruiz \& Pav., entre varias más conforme a la información a disposición (Tovar com. pers.).

Observaciones.- Salvia hunzikeri se alinea con el resto de especies de la sect. Flocculosae (Epling) Epling, integrado generalmente por sufrútices de ambientes secos o de marcada estacionalidad, con indumento típicamente ramificado, follaje más o menos caduco y microfilia a partir de renuevos axilares, inflorescencias terminales, flores de polinización entomófila con brácteas efímeras o persistentes, corolas celestes o azules con el tubo tan largo como el cáliz o apenas sobresaliente, interiormente sin papilas, estambres inclusos con dientes retrorsos en los vecciarios, estilo viloso y clusas mixogénicas.

Por su aspecto vegetativo, S. hunzikerii es superficialmente semejante a $S$. flocculosa Benth., que habita en Ecuador, debido a la forma y dimensiones de las láminas foliares; sus flores mayores y las brácteas tempranamente caducas impiden, no obstante, errores en la discriminación de ambas especies.

Claramente es reconocible entre las especies peruanas restantes de la sect. Flocculosae con pubescencia dendriforme al compararlas. Así, S. grisea Epling se diferencia básicamente en dos caracteres; uno fácil de advertir a simple vista: las láminas foliares oblongas -con bases truncado-cordadas y ápices obtusos-, que, además, se muestran conspicuamente ampollosas y discoloras; otro, que exige la observación paciente del cáliz: los pelos ramificados dendriformes sencillos, comunes a ambas especies, se entremezclan con algunos otros que muestran ramas glandulares. De la misma forma, son notorias sus diferencias con S. griseifolia Epling, que se aparta por sus brácteas florales persistentes durante la antesis y, al parecer, la fructificación y, a más de sus flores menores, también por sus hojas cortamente pecioladas con láminas angostamente ovadas y discoloras. Entre varios detalles, difiere de S. cruikshanksii Benth. y S. trifilis Epling por la forma de las hojas, que en ellas son ovadas a anchamente ovadas, además en la primera las láminas son usualmente glabrescentes o glabras cuando maduras mientras que en la segunda son discoloras con la superficie abaxial tomentosa; por otro lado, ambas se separan por sus flores de dimensiones menores. En tanto, S. xanthopylla Epling \& Játiva resulta poco afín por sus hojas sésiles con láminas angostamente oblongo-elípticas y márgenes revolutos, pero es su profusa pilosidad amarillenta, lanosa, que recubre casi toda la planta (excepto las corolas), el rasgo prominente que permite distinguirla y separarla fácilmente.

Por fin, podría confundirse con S. sarmentosa Epling del Perú pero el indumento en esta especie no está conformado por pelos ramificados, lo que de por sí constituye un atributo conspicuo y la hace fácil de separar.

Se ha señalado que, por lo común, la presencia de tricomas ramificados multicelulares reviste importancia taxonómica en la subfamilia Nepetoideae a nivel específico (Harley et al. 2004). En Salvia caracterizan, al menos en parte, a algunas secciones morfológicamente disímiles; lo que sugiere su evolución independiente en dichos grupos. Asimismo, otro rasgo de origen paralelo dentro del género en el Nuevo Mundo lo constituyen las formaciones ventrales en los brazos inferiores del conectivo que, a manera de dientes retrorsos, se presentan en $S$. hunzikeri y en el resto de la sect. Flocculosae. Claßen-Bockhoff et al. (2004) han apuntado, 
refiriéndose a S. rypara Briq. (sect. Malacophyllae Epling), que tales estructuras indudablemente están involucradas en el sistema de palanca de transferencia del polen, restringiendo el acceso al néctar y optimizando así la polinización por medio de abejas.

El hallazgo de tricomas dendriformes parcialmente capitados en un par de isotipos de S. grisea (Ferreyra 7628 MOL, USM) resulta interesante porque no habían sido mencionados en su descripción original (Epling \& Mathias 1957) ni tampoco reportados antes o con posteridad para ninguna otra especie peruana de Salvia (Epling 1938; Macbride 1960); empero, su constancia requiere confirmación, vista la escasez de materiales disponibles de la especie. Aquí se le atribuye tentativamente valor diagnóstico para distinguir S. grisea de S. hunzikeri.

La ausencia de mucílago en las clusas de $S$. hunzikeri es incierta y serán necesarios materiales adicionales de la especie para evaluar mejor tal carácter.

Ciertos especímenes: D.N. Smith 10575, D.N. Smith \& R. Valencia 10064, D.N. Smith et al. 9266 y 12038 (USM) y $A$. Granda \&J. Alegría 2438 (MOL), provenientes todos de Áncash, podrían señalarse, debido a cierta similitud en el follaje, como representantes de poblaciones geográficamente aisladas de S. hunzikeri pero son morfológicamente distintos por sus inflorescencias congestas, entre otros detalles, y constituyen una especie nueva no descrita.

\section{Agradecimientos}

Deseo expresar mi gratitud a Raymond M. Harley y Alan Paton por su colaboración; parte importante de la literatura y algunas fotografías de tipos nomenclaturales depositados en Kew los debo a su generosidad y buena disposición. Mi reconocimiento se hace extensivo a Philip Cantino, Susana Crespo, Maximilian Weigend, John R.I. Wood, Regine Claßen-Bockhoff y Petra Wester por facilitarme varias de las referencias aquí citadas. Dejo constancia de mi agradecimiento a John R.I. Wood y J. José Alegría por la lectura crítica y sus opiniones para mejorar los contenidos del texto; asimismo, a Marisa Ocrospoma, el entintado de la figura acompañante. Debo recordar a O. Tovar por prestar, con la cortesía que siempre lo caracterizó, algunos datos aquí consignados. Finalmente, agradezco a los directores y curadores de los herbarios MOL y USM por su amable apoyo.

\section{Literatura citada}

Claßen-Bockhoff R., M. Crone \& E. Baikovay. 2004. Stamen development in Salvia L.: homology reinvestigated. Int. J. Plant Sci. 165(4): 475-498.

Claßen-Bockhoff R., P. Wester \& E. Tweraser. 2003. The staminal lever mechanism in Salvia L. (Lamiaceae) - a review. Plant Biol. 5: 33-41.
Epling C. 1938. The Labiatae of Peru. Repert. Spec. Nov. Regni Veg. Beih. 105: 1-93.

Epling C. 1939. A revision of Salvia, subgenus Calosphace. Repert. Spec. Nov. Regni Veg. Beih. 110: 1-383.

Epling C. \& M.E. Mathias. 1957. Supplementary notes on American Labiatae - VI. Brittonia 8(4): 297-313.

Fernández-Alonso J.L. 2003. Estudios en Labiatae de Colombia IV. Novedades en Salvia y sinopsis de las secciones Angulatae y Purpureae. Caldasia 25(2): 235-281.

Harley R.M. 1992. Chromosome Numbers in Tropical American Labiatae. En: R.M. Harley \& T. Reynolds (eds.), Advances in Labiate Science. Royal Botanic Gardens, Kew. Pp. 211-246.

Harley R.M., S. Atkins, A.L. Budantsev, P.D. Cantino, B.J. Conn, R. Grayer, M.M. Harley, R. de Kok, T. Krestovskaja, R. Morales, A.J. Paton, O. Ryding \& T. Upson. 2004. Labiatae. En: J.W. Kadereit (ed.), The Families and Genera of Vascular Plants VII. Flowering Plants. Dycotiledons. Lamiales (except Acanthaceae including Avicenniaceae). Springer. Berlín. Pp. 167-275.

Macbride J.F. 1960. Labiatae. En: J.F. Macbride (ed.), Flora of Peru. Field Mus. Nat. Hist., Bot. Ser. 13, part 5(2): 721-829.

Meeuse A.D.J. 1992. Anthecology of the Labiatae: an Armchair Approach. En: R.M. Harley \& T. Reynolds (eds.), Advances in Labiate Science. Royal Botanic Gardens, Kew. Pp. 183-191.

Paton A. 1990. The phytogeography of Scutellaria L. Notes Roy. Bot. Gard. Edinburgh 46(3): 345-359.

Tovar O. 1957. Las gramíneas de Huancavelica. Primera parte. Mem. Mus. Hist. Nat. "Javier Prado" 6: 1-110.

Walker J.B. \& K.J. Sytsma. 2007. Staminal evolution in the genus Salvia (Lamiaceae): molecular phylogenetic evidence for multiple origins of the staminal lever. Ann. Bot. 100: 375-391.

Walker J.B., K.J. Sytsma, J. Treutlein \& M. Wink. 2004. Salvia (Lamiaceae) is not monophyletic: implications for the systematics, radiation, and ecological specializations of Salvia and tribe Mentheae. Amer. J. Bot. 91(7): 1115-1125.

Weberbauer A. 1945. El Mundo Vegetal de los Andes Peruanos. Editorial Lumen, Lima, Perú.

Wester P. \& R. Claßen-Bockhoff. 2007. Floral diversity and pollen transfer mechanisms in bird-pollinated Salvia species. Ann. Bot. 100: 401-421.

Wood J.R.I. 2007. The Salvias (Lamiaceae) of Bolivia. Kew Bull. 62(2): 177-222.

Wood J.R.I. \& R.M. Harley. 1989. The genus Salvia (Labiatae) in Colombia. Kew Bull. 44(2): 211-278. 